\title{
Clinical, behavioural and neural validation of the PANSS amotivation factor
}

Kaliuzhna, Mariia ; Kirschner, Matthias ; Carruzzo, Fabien ; Hartmann-Riemer, Matthias N ; Bischof, Martin ; Seifritz, Erich ; Tobler, Philippe N ; Kaiser, Stefan

\begin{abstract}
Negative symptoms in schizophrenia have been suggested to map onto two distinct factors - amotivation and diminished expression. Only recently, two-factor solutions for measuring negative symptoms have been proposed for the Positive and Negative Symptom Scale (PANSS), the most commonly used scale to assess the psychopathology of patients with schizophrenia. We aimed to validate the PANSS two-factor structure on a clinical, behavioural and neural level. For this multi-level validation, we reanalysed several datasets with patients for whom both the Brief Negative Symptom Assessment Scale (BNSS) and PANSS data were collected. We used a clinical dataset $(\mathrm{n}=120)$ as well as behavioural data from an effort-based decision making task $(\mathrm{n}=31)$ and functional neuroimaging data from a monetary incentive delay task $(n=41)$. Both tasks have previously been shown to be associated with BNSS amotivation. On the clinical level, the PANSS amotivation and diminished expression were highly correlated with their BNSS counterparts. On the behavioural level, we found that the PANSS amotivation factor but not the diminished expression factor specifically associated with willingness to invest effort to obtain a reward. On the neural level, PANSS amotivation was specifically related to reduced ventral striatal activation during reward anticipation. Our data confirm that the PANSS clearly allows distinguishing amotivation from diminished expression, as it relates selectively to specific aspects of behaviour and brain function. Our results will allow a re-analysis and sharing of existing datasets that used the PANSS to further substantiate the distinction between the two factors in neuroscientific studies and clinical trials.
\end{abstract}

DOI: https://doi.org/10.1016/j.schres.2020.04.018

Posted at the Zurich Open Repository and Archive, University of Zurich

ZORA URL: https://doi.org/10.5167/uzh-191588

Journal Article

Accepted Version

Originally published at:

Kaliuzhna, Mariia; Kirschner, Matthias; Carruzzo, Fabien; Hartmann-Riemer, Matthias N; Bischof, Martin; Seifritz, Erich; Tobler, Philippe N; Kaiser, Stefan (2020). Clinical, behavioural and neural validation of the PANSS amotivation factor. Schizophrenia Research, 220:38-45.

DOI: https://doi.org/10.1016/j.schres.2020.04.018 


\section{Clinical, behavioural and neural validation of the PANSS amotivation factor}

Mariia Kaliuzhna ${ }^{1}$, Matthias Kirschner ${ }^{2,3}$, Fabien Carruzzo ${ }^{1}$, Matthias N. Hartmann-Riemer ${ }^{2}$, Martin Bischof $^{2}$, Erich Seifritz ${ }^{2}$, Philippe N. Tobler ${ }^{4}$, Stefan Kaiser ${ }^{1,2}$

\footnotetext{
${ }^{1}$ Clinical and Experimental Psychopathology Group, Department of Psychiatry, University of Geneva

${ }^{2}$ Department of Psychiatry, Psychotherapy and Psychosomatics, Psychiatric Hospital, University of Zurich, Switzerland

${ }^{3}$ Montreal Neurological Institute, McGill University, Montreal, Canada

${ }^{4}$ Laboratory for Social and Neural Systems Research, Department of Economics, University of Zurich
}

Keywords: Schizophrenia; negative symptoms; amotivation; diminished expression; PANSS; BNSS 


\begin{abstract}
Negative symptoms in schizophrenia have been suggested to map onto two distinct factors - amotivation and diminished expression. Only recently, two-factor solutions for measuring negative symptoms have been proposed for the Positive and Negative Symptom Scale (PANSS), the most commonly used scale to assess the psychopathology of patients with schizophrenia. We aimed to validate the PANSS two-factor structure on a clinical, behavioural and neural level.

For this multi-level validation, we reanalysed several datasets with patients for whom both the Brief Negative Symptom Assessment Scale (BNSS) and PANSS data were collected. We used a clinical dataset $(n=120)$ as well as behavioural data from an effort-based decision making task $(n=31)$ and functional neuroimaging data from a monetary incentive delay task $(n=41)$. Both tasks have previously been shown to be associated with BNSS amotivation.

On the clinical level, the PANSS amotivation and diminished expression were highly correlated with their BNSS counterparts. On the behavioural level, we found that the PANSS amotivation factor but not the diminished expression factor specifically associated with willingness to invest effort to obtain a reward. On the neural level, PANSS amotivation was specifically related to reduced ventral striatal activation during reward anticipation.

Our data confirm that the PANSS clearly allows distinguishing amotivation from diminished expression, as it relates selectively to specific aspects of behaviour and brain function. Our results will allow a re-analysis and sharing of existing datasets that used the PANSS to further substantiate the distinction between the two factors in neuroscientific studies and clinical trials.
\end{abstract}




\section{Introduction}

According to a recent consensus, negative symptoms in schizophrenia are composed of five domains, namely, asociality, anhedonia, avolition, blunted affect, and alogia (Kirkpatrick et al., 2006). The bulk of previous research has studied the relationship between patients' behaviour, cognitive ability, brain function and response to treatment in relation to negative symptoms as a unitary construct. However, it has been suggested that at least two distinct factors constitute negative symptoms and the current DSM$V$ distinguishes between two subdomains: amotivation (including avolition, asociality and anhedonia) and diminished expression (including blunted affect and alogia) (Blanchard and Cohen, 2005, Kirkpatrick, 2014). This distinction appears important because the two factors relate to different aspects of behaviour and might require different medical and psychological treatment (Dowd et al., 2016, Gold et al., 2012) .

It is important to note that Strauss and colleagues provided strong statistical evidence for a five-factor model of negative symptoms corresponding to the five domains mentioned above (Ahmed et al., 2018, Strauss et al., 2018, San Ang et al., 2019). However, it remains to be determined which level of granularity is the most promising for understanding the pathophysiology of negative symptoms and developing new treatment approaches. At present, the two-factor approach has been successfully used in studies of behaviour and brain function, while less data are available for the five-factor approach (Kirschner et al., 2016, Hartmann et al., 2014, Moran et al., 2019, Waltz et al., 2018).

The most widely used scale for symptom assessment in research and clinical trials in schizophrenia is the Positive and Negative Symptom Scale (PANSS) (Kay et al., 1987). This scale allows a broad assessment of positive, negative and general symptoms. It has been argued, however, that the negative symptom subscale is ill-suited for specifically studying negative symptoms, as it disregards some items pertaining to negative symptoms (e.g. appetitive \& consummatory aspects of anhedonia) and includes items that relate to other symptom domains (e.g. stereotyped thinking, difficulty in abstract thinking) (Garcia-Portilla et al., 2015, Kirkpatrick et al., 2006). Importantly, two-factor solutions for negative symptoms have been described for the PANSS - (social) amotivation and diminished expression (Table 2) (Fervaha et al., 2014, Liemburg et al., 2013). These solutions include items from both the negative and the general subscales. It is unclear, however, how the two factors identified in the PANSS relate to the two factors of negative symptoms measured by specific negative symptom scales and whether they relate in a similar fashion to behaviour and brain function.

In order to assess the five domains of negative symptoms defined in the NIMH-Matrics consensus statement two newer specific scales were developed: the Brief Negative Symptom Assessment Scale (BNSS) (Strauss et al., 2012a, Strauss et al., 2012b) and the Comprehensive Assessment Interview for Negative Symptoms (CAINS) (Kring et al., 2013). While assessing the five identified domains of negative symptoms, both scales allow to define a two-factor structure, which has been found in several factor analytic studies (Mucci et al., 2015, Strauss et al., 2012a, Horan et al., 2011).

Importantly, recent work shows that amotivation and diminished expression as evaluated by the BNSS (but also by the Scale for the Assessment of Negative Symptoms (SANS) (Andreasen, 1982) relate differentially to several aspects of behaviour and have different underlying neural substrates. For example, amotivation, but not diminished expression, has been associated with deficits in reward learning and effort-based decision-making (Hartmann et al., 2014, Hartmann et al., 2015, Gold et al., 2012, Waltz et al., 2007, Raffard et al., 2016, Grant and Beck, 2008). On the other hand, diminished expression, but not amotivation, has been associated with abnormalities in emotion perception and in the execution and 
imitation of facial expressions (Gur et al., 2006, Lepage et al., 2011). However, these findings result from a limited number of studies with mostly small sample sizes and have therefore still to be considered with caution.

In the present manuscript, we aimed for the first time to validate the PANSS two-factor structure not only on the clinical but also on a behavioural and neural level. Such a novel multilevel validation would grant neuroscientific validity to the concept of amotivation, linking clinical observations of negative symptoms to pathophysiology. This confirmation of the PANSS two-factor structure in turn would allow a fine-grained re-analysis of the existing data on pathophysiology as well as treatment of negative symptoms and would facilitate including factor scores in planned trials.

Specifically, we relied on several datasets with patients for whom both BNSS and PANSS data were collected and we tested the following hypotheses (see Methods section for detailed information on the patient cohorts). First, if indeed the factors amotivation and diminished expression as measured by the two scales represent the same phenomena, we should observe stronger correlations between the same factors (e.g. BNSS amotivation \& PANSS amotivation) than between the different factors (e.g. BNSS amotivation \& PANSS diminished expression). Second, using the PANSS amotivation measure we should replicate the previously reported (Hartmann et al., 2015) correlation between amotivation (as measured by BNSS) and the discounting of reward when physical effort is required. Moreover, this relation should be specific for amotivation rather than diminished expression. Third, following on from our neural findings (Kirschner et al., 2016, Stepien et al., 2018), we expected to observe a negative correlation of ventral striatum activity during reward anticipation with the amotivation, but not the diminished expression factor when using the PANSS instead of the BNSS.

\section{Methods}

\section{Psychopathological assessment and factor models used in all studies}

In all patients, the diagnosis of schizophrenia or schizoaffective disorder was confirmed using the MiniInternational Neuropsychiatric Interview for DSM-IV (MINI). Exclusion criteria comprised: presence of other DSM-IV Axis I disorders (in particular, current substance use disorder and major depressive disorder); presence of florid psychotic symptomatology (i.e., a score exceeding 4 on the positive subscale of the PANSS); presence of extrapyramidal side-effects (total score exceeding 2 on the Modified Sympson-Angus Scale) and medication with more than $1 \mathrm{mg}$ lorazepam per day. These exclusion criteria aimed at limiting the impact of secondary negative symptoms on the present results (Kirschner et al., 2017).

Negative symptoms were assessed with the BNSS, the PANSS and the SANS. The BNSS served as the primary clinical outcome for the present studies. The validated German version of the scale was employed. All raters were trained by the senior author (SK) who was responsible for the German translation.

In order to distinguish between the factors amotivation and diminished expression we used the existing factor solutions from the literature for the three scales (Table 2). For the BNSS, we adopted the two-factor solution proposed by Mucci and colleagues (2015), which excludes the item "lack of normal distress". For the PANSS we used the factor structure proposed by Fervaha and colleagues (2014) that does not include two additional items included in the solution of Liemburg and colleagues (2013) (see discussion for more detail). However, the results of all analyses conducted were similar for the two solutions. 
In the main manuscript, we focus on the PANSS and the BNSS. However, to provide a complete overview we also performed all analyses with a two-factor solution for the SANS (Strauss et al., 2013). The results for the SANS can be found in the Supplementary material, and closely resemble those for the BNSS and the PANSS.

\section{Study 1 - Clinical validation}

The goal of the first study was to validate the amotivation - diminished expression distinction in the PANSS don a clinical level.

\section{Participants}

Patients' demographics are presented in Table 1. Data from 120 patients from different studies were used to increase power. 75 patients are from the study by Bischof et al., 2016 (Bischof et al., 2016), data from 31 of these patients were used in Study 2 of this manuscript, and data of 27 patients in Study 3.

Data from the remaining 45 patients were collected as part of a yet unpublished study in our laboratory. Thus, 75 data points were only used in Study 1 and do not overlap with the other two studies presented in this manuscript.

110 patients fulfilled the Diagnostic and Statistical Manual of Mental Disorders, Fourth Edition (DSM-IV) criteria for schizophrenia and 10 - for schizoaffective disorder (not in a mood episode at the time of testing). 73 patients of this cohort were at the end of their hospitalisation and 47 were outpatients.

\section{Statistical analyses}

Jamovi was used to perform the correlations (The jamovi project (2019), jamovi (Version 0.9). We conducted Pearson correlational analyses between BNSS and PANSS factors using the ratings of all 120 participants. In addition, we performed the same analysis correlating PANSS and SANS factors using data from the 75 participants for whom SANS scores were available (see supplementary material). We used the cocor R package to compare the strengths of the correlations (Diedenhofen and Musch, 2015). The significance level was set to $p=0.05$.

\section{Study 2 - Behavioural validation}

The second study aimed to validate the PANSS factor structure on a behavioural level. For this purpose, we employed data from Hartmann and colleagues (2015), which showed a negative correlation between willingness to exert effort and the factor amotivation of the BNSS. We used the same analytic approach, but used the PANSS factors instead of the BNSS factors.

Participants 
Data from the original 31 patients were used for the analysis (Table 1). Patients met the DSM-IV diagnostic criteria for schizophrenia $(n=25)$ or schizoaffective disorder $(n=6)$ (confirmed by the MINI). All patients were clinically and pharmacologically stable at the time of the study. The same exclusion criteria as for Study 1 were applied. 27 patients were part of the Study 1 cohort, namely, their data represents a subset of the 75 patients from the work of Bischof et al., 2016, and 4 patients did not overlap with the other studies of this manuscript.

\section{Effort task}

The task consisted of a series of choices between two options: receiving a small default amount of money (1 CHF) while exerting no effort versus receiving a larger amount (e.g. $3 \mathrm{CHF}$ ) contingent on the exertion of a certain level of physical effort. Physical effort involved squeezing a hand-held dynamometer with the dominant hand during a 3.5s interval (in the no-effort choice participants had to wait for 3.5s before receiving the reward). The effortful choice comprised one of four effort levels: $40 \%, 60 \%, 80 \%$ and $100 \%$ of maximal strength. Maximal strength was determined at the beginning of the experiment in a calibration trial, where participants had to press the handgrip as hard as they could. The following amounts constituted the reward on effortful trials: $1.5,2,2.5,3,5 \mathrm{CHF}(1 \mathrm{CHF} \approx 1 \$$ ). Participants performed 80 trials (each effort level was repeated 4 times).

This task allows estimating the reward at which participants are indifferent between a no-effort choice and a particular effortful option (i.e. indifference points) by fitting a logistic function through the fraction of all effortful choices for a given reward level. The indifference points further allow calculating the relative subjective value (SV) of reward for each participant, that is, how the four effort levels reduce the value of a given reward. SV is calculated by dividing the default reward of $1 \mathrm{CHF}$ by the respective indifference reward amounts. Finally, connecting SVs over the four effort levels we used the area under the curve (AUC) as a single measure of willingness to exert effort. Smaller AUC corresponds to steeper effort discounting.

\section{Statistical Analyses}

The present analysis aimed to replicate the original behavioural results obtained with the BNSS. The authors correlated the AUC with the amotivation and diminished expression factors of the BNSS. We thus performed the correlational analysis on the original data of this study (AUC) but used the two factors from the PANSS and SANS scores, collected at the time of the study.

\section{Study 3 - Neural validation}

The third analysis aimed to validate the PANSS factor structure on the neural level. For this purpose, we employed data from the studies by Kirschner and colleagues (2016), and by Stepien and colleagues (2018), which showed a negative correlation between ventral striatal activation during reward anticipation and the factor amotivation of the BNSS. We used the same analytic approach, but used the PANSS factors instead of the BNSS factors. Since the main objective of the present analysis concerns the neural validation of the PANSS factors, we focussed on ventral striatal activation as the best established neural correlate of amotivation and did not perform a whole-brain analysis. 


\section{Participants}

27 clinically stable patients fulfilling the diagnostic criteria of DSM-IV for schizophrenia participated in the study by Kirschner and colleagues (2016). These patients were also part of the 75 patients (Bischof et al., 2016) from Study 1 and partially overlapped with patients in Study 2 (9 patients were the same). Additional data from 16 patients with schizophrenia were included. These patients originally participated in the study by Stepien and colleagues (2018). These patients did not overlap with any patients from Study 1 or 2 . Two patients from the Kirschner et al. study also participated in the Stepien et al. study. We excluded their data for the study by Kirschner and colleagues (2016). Thus, data from 41 participants was analysed.

\section{Task design}

In both studies, participants performed a version of the Monetary Incentive Delay Task (MID) originally developed by Simon and colleagues (Simon et al., 2015). At the beginning of each trial, a cue informed the participants of the maximal amount they could win in that trial (0 CHF, $0.40 \mathrm{CHF}$ or $2 \mathrm{CHF}$ ). Next, participants had to identify an outlier from three circular stimuli as fast and as correctly as possible. Feedback on how much they won was presented on every trial and depended on the speed of the participants' response. There was a total of 72 trials in the experiment.

\section{Functional Magnetic Resonance Imaging}

Imaging was performed at the MR Zentrum of the Psychiatric Hospital, University of Zurich, using a Philips Achieva 3.0 T magnetic resonance scanner with a 32-channel SENSE head coil. The task was conducted in two runs, 195 images in each run. A gradient-echo $T_{2}{ }^{*}$-weighted echo-planar image (EPI) sequence was used with 38 slices acquired in ascending order. Acquired in-plane resolution was $3 \times 3 \mathrm{~mm}^{2}, 3 \mathrm{~mm}$ slice thickness and $0.5 \mathrm{~mm}$ gap width over a field of view of $240 \times 240 \mathrm{~mm}$, repetition time $2000 \mathrm{~ms}$, echo time $25 \mathrm{~ms}$ and a flip angle of $82^{\circ}$. The first 5 scans were discarded to eliminate the influence of $T_{1}$ saturation effects. Slices were aligned with the anterior-posterior commissure. Anatomical data were acquired using an ultrafast gradient echo $T_{1}$-weighted sequence in 160 sagittal plane slices of $240 \times 240 \mathrm{~mm}$ resulting in $1 \times 1 \times 1 \mathrm{~mm}$ voxels.

\section{Analyses}

fMRI analyses were performed with SPM8. During preprocessing functional images were corrected for differences in the time of slice acquisition and motion using the realign and unwarp functions of SPM8. Correction for combined static and dynamic distortions was done using a voxel displacement map, calculated from double phase and magnitude field map data. Segmentation, bias correction and spatial normalization was performed. Images were smoothed using a $6 \mathrm{~mm}$ full-width at half-maximum Gaussian kernel. Participants with translational head movement greater than $3 \mathrm{~mm}$ or extensive signal dropout in the EPI sequences were excluded to ensure adequate data quality.

For the first level analysis, a general linear model (GLM) approach was used in an event-related design. Three separate regressors were used for the anticipation phases of the three reward levels: anticipation of no reward $(0 \mathrm{CHF})$, low reward $(0.40 \mathrm{CHF})$ and high reward $(2 \mathrm{CHF})$. Similarly, 3 regressors were included for the outcome phase. For the low and high reward conditions the outcome regressors were 
parametrically modulated by the amount the participants won on each trial. Target presentation and error anticipation, target and outcome phase were modelled as regressors of no interest. All explanatory variables were convolved using the canonical hemodynamic response function. The contrast "high reward (2 CHF) anticipation versus no reward (0 CHF) anticipation" was calculated for reward anticipation.

The anticipation regressor of interest included the cue and the delay phases of each trial. Thus, as mentioned above, there were three regressors of interest (anticipation of $0 \mathrm{CHF}, 0.40 \mathrm{CHF}$ or $2 \mathrm{CHF}$ ). Based on a meta-analysis (Knutson and Greer, 2008), the ventral striatum was defined as the region of interest during reward anticipation (left: $x, y, z=-12,10,-2$; right: $x, y, z=10,8,0$ ). Mean percent signal change was extracted with the REX toolbox (http://web.mit.edu/swg/software.htm). The data from the two studies were pooled and correlations between the percent signal change in the ventral striatum and the factors amotivation and diminished expression of the BNSS and the PANSS were calculated.

\section{Results}

\section{Study 1 - Clinical Validation}

Comparing the two PANSS factors to the two BNSS factors

As predicted by the first hypothesis, we found that the PANSS amotivation factor was highly correlated with the BNSS amotivation factor (Figure 1A). Importantly, this correlation was significantly stronger than the correlation between the PANSS amotivation factor and the BNSS diminished expression score $(z=5.2$, $p<0.0001)$. Furthermore, the PANSS diminished expression factor was highly correlated with the BNSS diminished expression factor (Figure 1D) and this correlation was significantly stronger than the correlation between the PANSS diminished expression factor and the BNSS amotivation factor ( $z=5.4$, $p<0.0001)$. Thus, the two-factor solutions of the two scales converged, suggesting that they measure amotivation and diminished expression similarly.

In an additional analysis, we included only the 75 patients which exclusively participated in Study 1 and not in Study 2 or 3 in order assure full independence. The results for this subgroup were almost identical to the full sample (see supplementary material, and supplementary table S5).

Comparing the PANSS amotivation factor to the three sub-domains of BNSS amotivation

While our main hypotheses concerned the correspondence between the PANSS and BNSS two-factor model, we were also interested in the association of the PANSS two-factor model with the BNSS five-factor model (see Table 4 \& 5). PANSS amotivation was highly and significantly correlated with each of the three sub-domains composing BNSS amotivation, namely, anhedonia, asociality and avolition (the correlation between PANSS amotivation and BNSS asociality was significantly stronger than that between PANSS amotivation and BNSS anhedonia $(z=-2.0, p=0.045))$. Similarly, PANSS diminished expression correlated significantly with BNSS blunted affect and alogia.

\section{Study 2 - Behavioural validation}

Correlation between effort discounting and amotivation 
We next performed correlations between the willingness to exert effort (AUC) from the study by Hartmann and colleagues (2015) and the amotivation factors extracted from the different negative symptoms scales (Table 6, Figure 2). The original study (Hartmann et al., 2015) found a significant negative correlation between AUC and the factor amotivation of the BNSS $(r=-0.67, p<0.0001)$ but no significant correlation with the diminished expression factor $(r=-0.14, p=0.45)$. Thus, patients who had more amotivation symptoms discounted physical effort more strongly. In other words, patients with higher amotivation scores were less willing to work for reward in the behavioural task.

In the present analysis we found a highly significant negative correlation between the willingness to exert effort and the PANSS amotivation factor (PANSS: $r=-0.49, p=0.005$ ). There was no significant correlation with the PANSS diminished expression factor (PANSS: $r=-0.08, p=0.68$ ). The two correlations were significantly different from each other $(z=-3.0, p=0.003)$. Thus, the pattern of results is similar to the one obtained for the BNSS factors. However, the size of the correlation with PANSS amotivation was significantly smaller than with BNSS amotivation $(z=-2.1, p=0.036)$. Thus, at the behavioural level the two scales appear to relate similarly to an independent measure of motivation, in line with the second hypothesis.

\section{Study 3 - Neural validation}

\section{Correlation between ventral striatal signal change and amotivation}

Our previous work has shown that activity in the ventral striatum during reward anticipation is negatively correlated with the amotivation but not the diminished expression factor of the BNSS (Kirschner et al., 2016, Stepien et al., 2018). We pooled the patients in these two studies in order to test the relation of PANSS and SANS to the signal change in the left ventral striatum (hypothesis 3 ).

In the pooled dataset, as expected, left ventral striatal activation showed a highly significant negative association with BNSS amotivation $(r=-0.53, p<0.001)$, but not with BNSS diminished expression $(r=0.05$, $\mathrm{p}=0.78)$. Corroborating hypothesis 3 , left ventral striatal activation was negatively associated with PANSS amotivation ( $r=-0.33, p=0.04)$ but not with PANSS diminished expression $(r=0.009, p=0.96)$ and the difference between these correlations was significant $(z=-2.5, p=0.013)$. Thus, similar to BNSS the PANSS factors have distinct relationships to left ventral striatum signal change during reward anticipation. However, left ventral striatum signal correlated numerically and trend-level statistically $(z=-1.9, p=0.053)$ more negatively with BNSS amotivation than with PANSS amotivation.

\section{Discussion}

The goal of the present paper was to evaluate whether the scale most commonly used in the study of schizophrenia - the PANSS - allows maintaining the two-factor distinction between amotivation and diminished expression similarly to the more recent and more complete BNSS. We conducted three correlational analyses on data at three processing levels - symptomatic, behavioural and neural, which showed that this was indeed the case, although the association between PANSS amotivation and its behavioural and neural correlates was weaker than for BNSS amotivation. 
First, on the clinical level, the correlations between BNSS and PANSS factors were significantly stronger within the same factor (within amotivation for BNSS and PANSS and within diminished expression for BNSS and PANSS) than across the two factors (e.g. BNSS amotivation and PANSS diminished expression). Thus, despite the differences between the scales, by following the factor-analytic structure defined in previous research, we show that BNSS and PANSS factors reflect similar symptom clusters.

Next, we show that the PANSS amotivation factor, but not the diminished expression factor, is specifically associated with reward discounting for physical effort. The observed effect size is smaller when using PANSS amotivation than the amotivation factor from the BNSS - a more specific negative symptom scale. The same differential result is obtained using the SANS amotivation / diminished expression factors.

Further, in an enlarged sample we confirm that reduced left ventral striatal signal change during reward anticipation is selectively associated with PANSS amotivation, but not with diminished expression (Kirschner et al., 2016, Stepien et al., 2018). A similar result is observed when using the SANS. In line with the results for the effort discounting task, the observed effect size for the association with ventral striatal signal change is somewhat smaller when using PANSS amotivation than with the two specific negative symptom scales BNSS and SANS.

The behavioural and neuroimaging results clearly show that the PANSS allows disentangling specific correlates for amotivation in comparison to diminished expression. Thus, they confirm the specific association shown on the clinical level and support the use of the PANSS for measuring the two symptom dimensions of amotivation and diminished expression. These results are in line with recent work showing an association of the PANSS amotivation factor with reduced ventral striatum activity and reduced effortful behaviour (Caravaggio et al., 2018, Chang et al., 2019). The fact that the observed effect size for the associations with PANSS amotivation is somewhat smaller than with the BNSS can be explained by the restriction of the PANSS to social amotivation, which does not cover the full range of amotivation symptoms. This is evidenced by the stronger correlation between PANSS amotivation and BNSS asociality (which corresponds to social amotivation) than between PANSS amotivation and BNSS anhedonia, the latter not being measured by the PANSS. The BNSS, on the other hand, explores motivation for social, work/study/therapy and leisure activities. Despite the reduced range of motivation explored by the PANSS it shows associations with behaviour and brain function that are comparable to the BNSS.

Several factor solutions exist for the PANSS. The two-factor solutions for the assessment of negative symptoms differ in that Liemburg and colleagues (2013) include items G5 (mannerisms and posturing) and G13 (avolition) as part of the diminished expression factor, whereas Fervaha and colleagues (2014) do not. We have used this latter solution, as it is more balanced in terms of number of elements per factor and because G5 and G13 are not regarded as negative symptoms per se according to the NIMH-Matrics consensus statement. Indeed, G13, even though bearing the name "avolition", clinically refers more to ambivalence and thus does not correspond to any item of the BNSS. In any case, using the PANSS factor solution by Liemburg and colleagues did not change the results of the present study in any meaningful way.

Our results indicate that large-scale studies not primarily focusing on negative symptoms can yield valuable insights on amotivation and diminished expression while only employing the PANSS, as opposed to a specialised negative symptom scale like the BNSS or the CAINS. However, as mentioned earlier, the PANSS remains constrained in the way different aspects of negative symptoms are addressed. PANSS mainly evaluates behavioural aspects of patients' symptoms and neglects internal experience. Similarly, 
PANSS does not measure anhedonia and the definition of avolition does not correspond to its current conceptualization (Kirkpatrick et al., 2006). It is thus crucial that specialised scales like the BNSS be used in studies directly addressing negative symptoms in order to obtain fine-grained specific results. Importantly, recent work has suggested that the five domains of negative symptoms represent distinct constructs and should be studied separately (Strauss et al., 2018, Ahmed et al., 2018). The PANSS does not allow this supplementary level of granularity, only afforded by specialised scales. The present data highlight the value of the PANSS in the study of negative symptoms. However, the BNSS, and similar scales, remain the clinical instruments of choice for a detailed study of this domain of psychopathology.

The tasks used in the present studies did not aim to show specific behavioural and neural correlates for the diminished expression factor. So far, a comprehensive model of diminished expression has not been established in the literature and empirical data are generally more limited (Kaiser et al., 2017). To date, several studies indicate that the amotivation factor is primarily related to functional outcomes (Chang et al., 2016, Fervaha et al., 2015), and therefore could be thought of as more relevant and a central target for the development of treatment interventions. However, previous work (Galderisi et al., 2013, Evensen et al., 2012), and most importantly, recent studies in large cohorts have found diminished expression to be linked to at least some aspects of community functioning (Liemburg et al., 2020). Furthermore, two recent studies needing confirmation have suggested diminished expression to respond in a similar fashion to pharmacological treatment interventions (Fleischhacker et al., 2019, Harvey et al., 2020) as amotivation. Our findings suggest that a re-analysis of existing behavioural and neuroimaging studies using the PANSS factor model could provide additional empirical data for these observations, allowing to develop a model of diminished expression.

The present studies have several limitations. First, all ratings (PANSS, BNSS, SANS) were based on the same semi-structured interview for negative symptoms, which was modified from the BNSS manual. Thus, we cannot be certain that in a study that only uses the PANSS the distinction between factors would be as clear as in the present study. However, our results are coherent with previous factor analytic studies (Liemburg et al., 2013; Fervaha et al., 2014). Second, all ratings for one patient were made by the same rater, which is likely to increase correspondence between the different scales.

Overall, our results demonstrate how behavioural and neuroimaging data can provide an additional validation of clinical measures. To our knowledge, our study is the first to apply this novel approach to negative symptoms. We consider the mutual informing of clinical and neuroscientific approaches as important, as future clinical scales may not only be reliable and valid at a clinical level, but also link to the underlying pathophysiology. This multilevel approach is in line with the RDoC framework, the goal of which is to establish a neuroscience-driven classification of psychiatric disorders as opposed to one that is only based on clinical observations (Insel, 2014, Insel et al., 2010, Cuthbert and Insel, 2013).

Our results provide a building block for evaluating the extensive previous behavioural and brain imaging research using the PANSS in terms of selective relations to amotivation or diminished expression domains of negative symptoms. This approach is particularly valuable, as it would allow constituting data sets with large sample sizes increasing the power to detect the mechanisms underlying negative symptoms. These methodological results also promote the development of open science, making it possible to share data in order to construct such large samples. Thus, reliably linking the different domains of negative symptoms with specific pathophysiological mechanisms, such as impairments in reward processing, may create specific targets for biological and psychosocial interventions. 


\section{References}

AHMED, A. O., KIRKPATRICK, B., GALDERISI, S., MUCCI, A., ROSSI, A., BERTOLINO, A., ROCCA, P., MAJ, M., KAISER, S. \& BISCHOF, M. 2018. Cross-cultural validation of the 5 -factor structure of negative symptoms in schizophrenia. Schizophrenia bulletin, 45, 305-314.

ANDREASEN, N. C. 1982. Negative symptoms in schizophrenia: definition and reliability. Archives of general psychiatry, 39, 784-788.

BISCHOF, M., OBERMANN, C., HARTMANN, M. N., HAGER, O. M., KIRSCHNER, M., KLUGE, A., STRAUSS, G. P. \& KAISER, S. 2016. The brief negative symptom scale: validation of the German translation and convergent validity with self-rated anhedonia and observer-rated apathy. BMC psychiatry, 16, 415.

BLANCHARD, J. J. \& COHEN, A. S. 2005. The structure of negative symptoms within schizophrenia: implications for assessment. Schizophrenia bulletin, 32, 238-245.

CARAVAGgIO, F., FERVAHA, G., IWATA, Y., PLITMAN, E., CHUNG, J. K., NAKAJIMA, S., MAR, W., GERRETSEN, P., KIM, J. \& CHAKRAVARTY, M. M. 2018. Amotivation is associated with smaller ventral striatum volumes in older patients with schizophrenia. International journal of geriatric psychiatry, 33, 523-530.

CHANG, W. C., CHU, A. O. K., TREADWAY, M. T., STRAUSS, G. P., CHAN, S. K. W., LEE, E. H. M., HUI, C. L. M., SUEN, Y. N. \& CHEN, E. Y. H. 2019. Effort-based decision-making impairment in patients with clinically-stabilized first-episode psychosis and its relationship with amotivation and psychosocial functioning. European Neuropsychopharmacology, 29, 629-642.

CHANG, W. C., HUI, C. L. M., CHAN, S. K. W., LEE, E. H. M. \& CHEN, E. Y. H. 2016. Impact of avolition and cognitive impairment on functional outcome in first-episode schizophrenia-spectrum disorder: a prospective one-year follow-up study. Schizophrenia research, 170, 318-321.

CUTHBERT, B. N. \& INSEL, T. R. 2013. Toward the future of psychiatric diagnosis: the seven pillars of RDoC. BMC medicine, 11, 126.

DIEDENHOFEN, B. \& MUSCH, J. 2015. cocor: A comprehensive solution for the statistical comparison of correlations. PloS one, 10, e0121945.

DOWD, E. C., FRANK, M. J., COLLINS, A., GOLD, J. M. \& BARCH, D. M. 2016. Probabilistic reinforcement learning in patients with schizophrenia: Relationships to anhedonia and avolition. Biological psychiatry: cognitive neuroscience and neuroimaging, 1, 460-473.

EVENSEN, J., RøSSBERG, J. I., BARDER, H., HAAHR, U., TEN VELDEN HEGELSTAD, W., JOA, I., JOHANNESSEN, J. O., LARSEN, T. K., MELLE, I. \& OPJORDSMOEN, S. 2012. Flat affect and social functioning: a 10 year follow-up study of first episode psychosis patients. Schizophrenia research, 139, 99-104.

FERVAHA, G., FOUSSIAS, G., AGID, O. \& REMINGTON, G. 2014. Motivational and neurocognitive deficits are central to the prediction of longitudinal functional outcome in schizophrenia. Acta Psychiatrica Scandinavica, 130, 290-299.

FERVAHA, G., FOUSSIAS, G., AGID, O. \& REMINGTON, G. 2015. Motivational deficits in early schizophrenia: prevalent, persistent, and key determinants of functional outcome. Schizophrenia research, 166, 9-16. 
FLEISCHHACKER, W., GALDERISI, S., LASZLOVSZKY, I., SZATMÁRI, B., BARABÁSSY, Á., ACSAI, K., SZALAI, E., HARSÁNYI, J., EARLEY, W. \& PATEL, M. 2019. The efficacy of cariprazine in negative symptoms of schizophrenia: Post hoc analyses of PANSS individual items and PANSS-derived factors. European Psychiatry, 58, 1-9.

GALDERISI, S., BUCCI, P., MUCCI, A., KIRKPATRICK, B., PINI, S., ROSSI, A., VITA, A. \& MAJ, M. 2013. Categorical and dimensional approaches to negative symptoms of schizophrenia: focus on longterm stability and functional outcome. Schizophrenia research, 147, 157-162.

GARCIA-PORTILLA, M. P., GARCIA-ALVAREZ, L., SAIZ, P. A., AL-HALABI, S., BOBES-BASCARAN, M. T., BASCARAN, M. T., MUÑIZ, J. \& BOBES, J. 2015. Psychometric evaluation of the negative syndrome of schizophrenia. European archives of psychiatry and clinical neuroscience, 265, 559566.

GOLD, J. M., WALTZ, J. A., MATVEeVA, T. M., KASANOVA, Z., STRAUSS, G. P., HERBENER, E. S., COLLINS, A. G. \& FRANK, M. J. 2012. Negative symptoms and the failure to represent the expected reward value of actions: behavioral and computational modeling evidence. Archives of general psychiatry, 69, 129-138.

GRANT, P. M. \& BECK, A. T. 2008. Defeatist beliefs as a mediator of cognitive impairment, negative symptoms, and functioning in schizophrenia. Schizophrenia bulletin, 35, 798-806.

GUR, R. E., KOHLER, C. G., RAGLAND, J. D., SIEGEL, S. J., LESKO, K., BILKER, W. B. \& GUR, R. C. 2006. Flat affect in schizophrenia: relation to emotion processing and neurocognitive measures. Schizophrenia bulletin, 32, 279-287.

HARTMANN, M. N., HAGER, O. M., REIMANN, A. V., CHUMBLEY, J. R., KIRSCHNER, M., SEIFRITZ, E., TOBLER, P. N. \& KAISER, S. 2014. Apathy but not diminished expression in schizophrenia is associated with discounting of monetary rewards by physical effort. Schizophrenia bulletin, 41, 503-512.

HARTMANN, M. N., KLUGE, A., KALIS, A., MOJZISCH, A., TOBLER, P. N. \& KAISER, S. 2015. Apathy in schizophrenia as a deficit in the generation of options for action. Journal of abnormal psychology, 124, 309.

HARVEY, P. D., SAOUD, J. B., LUTHRINGER, R., MOROZ, S., BLAZHEVYCH, Y., STEFANESCU, C. \& DAVIDSON, M. 2020. Effects of Roluperidone (MIN-101) on two dimensions of the negative symptoms factor score: Reduced emotional experience and reduced emotional expression. Schizophrenia Research, 215, 352-356.

HORAN, W. P., KRING, A. M., GUR, R. E., REISE, S. P. \& BLANCHARD, J. J. 2011. Development and psychometric validation of the Clinical Assessment Interview for Negative Symptoms (CAINS). Schizophrenia research, 132, 140-145.

INSEL, T., CUTHBERT, B., GARVEY, M., HEINSSEN, R., PINE, D. S., QUINN, K., SANISLOW, C. \& WANG, P. 2010. Research domain criteria (RDoC): toward a new classification framework for research on mental disorders. Am Psychiatric Assoc.

INSEL, T. R. 2014. The NIMH research domain criteria (RDoC) project: precision medicine for psychiatry. American Journal of Psychiatry, 171, 395-397.

KAISER, S., LYNE, J., AGARTZ, I., CLARKE, M., MØRCH-JOHNSEN, L. \& FAERDEN, A. 2017. Individual negative symptoms and domains-relevance for assessment, pathomechanisms and treatment. Schizophrenia research, 186, 39-45.

KAY, S. R., FISZBEIN, A. \& OPLER, L. A. 1987. The positive and negative syndrome scale (PANSS) for schizophrenia. Schizophrenia bulletin, 13, 261-276.

KIRKPATRICK, B. 2014. Developing concepts in negative symptoms: primary vs secondary and apathy vs expression. The Journal of clinical psychiatry.

KIRKPATRICK, B., FENTON, W. S., CARPENTER, W. T. \& MARDER, S. R. 2006. The NIMH-MATRICS consensus statement on negative symptoms. Schizophrenia bulletin, 32, 214-219. 
KIRSCHNER, M., HAGER, O. M., BISCHOF, M., HARTMANN, M. N., KLUGE, A., SEIFRITZ, E., TOBLER, P. N. \& KAISER, S. 2016. Ventral striatal hypoactivation is associated with apathy but not diminished expression in patients with schizophrenia. Journal of psychiatry \& neuroscience: JPN, 41, 152.

KNUTSON, B. \& GREER, S. M. 2008. Anticipatory affect: neural correlates and consequences for choice. Philosophical Transactions of the Royal Society B: Biological Sciences, 363, 3771-3786.

KRING, A. M., GUR, R. E., BLANCHARD, J. J., HORAN, W. P. \& REISE, S. P. 2013. The clinical assessment interview for negative symptoms (CAINS): final development and validation. American Journal of Psychiatry, 170, 165-172.

LEPAGE, M., SERGERIE, K., BENOIT, A., CZECHOWSKA, Y., DICKIE, E. \& ARMONY, J. 2011. Emotional face processing and flat affect in schizophrenia: functional and structural neural correlates. Psychological medicine, 41, 1833-1844.

LIEMBURG, E., CASTELEIN, S., STEWART, R., VAN DER GAAG, M., ALEMAN, A., KNEGTERING, H. \& INVESTIGATORS, O. O. P. 2013. Two subdomains of negative symptoms in psychotic disorders: established and confirmed in two large cohorts. Journal of psychiatric research, 47, 718-725.

LIEMBURG, E. J., ENRIQUEZ-GEPPERT, S., WARDENAAR, K. J., BRUGGEMAN, R., ALEMAN, A., CASTELEIN, S., KNEGTERING, H., VELING, W., ALIZADEH, B. Z. \& VAN AMELSVOORT, T. 2020. Expressive deficits and amotivation as mediators of the associations between cognitive problems and functional outcomes: Results from two independent cohorts. Schizophrenia Research.

MORAN, E., CULBRETH, A., KANDALA, S. \& BARCH, D. 2019. From neuroimaging to daily functioning: A multimethod analysis of reward anticipation in people with schizophrenia. Journal of abnormal psychology.

MUCCI, A., GALDERISI, S., MERLOTTI, E., ROSSI, A., ROCCA, P., BUCCI, P., PIEGARI, G., CHIEFFI, M., VIGNAPIANO, A. \& MAJ, M. 2015. The Brief Negative Symptom Scale (BNSS): independent validation in a large sample of Italian patients with schizophrenia. European Psychiatry, 30, 641647.

RAFFARD, S., GUTIERREZ, L.-A., YAZBEK, H., LARUE, A., BOULENGER, J.-P., LANÇON, C., BENOIT, M., FAGET, C., NORTON, J. \& CAPDEVIELLE, D. 2016. Working memory deficit as a risk factor for severe apathy in schizophrenia: a 1-year longitudinal study. Schizophrenia bulletin, 42, 642-651.

SAN ANG, M., REKHI, G. \& LEE, J. 2019. Validation of the Brief Negative Symptom Scale and its association with functioning. Schizophrenia research, 208, 97-104.

SIMON, J. J., CORDEIRO, S. A., WEBER, M.-A., FRIEDERICH, H.-C., WOLF, R. C., WEISBROD, M. \& KAISER, S. 2015. Reward system dysfunction as a neural substrate of symptom expression across the general population and patients with schizophrenia. Schizophrenia bulletin, 41, 1370-1378.

STEPIEN, M., MANOLIU, A., KUBLI, R., SCHNEIDER, K., TOBLER, P. N., SEIFRITZ, E., HERDENER, M., KAISER, S. \& KIRSCHNER, M. 2018. Investigating the association of ventral and dorsal striatal dysfunction during reward anticipation with negative symptoms in patients with schizophrenia and healthy individuals. PloS one, 13, e0198215.

STRAUSS, G. P., HONG, L. E., GOLD, J. M., BUCHANAN, R. W., MCMAHON, R. P., KELLER, W. R., FISCHER, B. A., CATALANO, L. T., CULBRETH, A. J. \& CARPENTER, W. T. 2012a. Factor structure of the brief negative symptom scale. Schizophrenia research, 142, 96-98.

STRAUSS, G. P., HORAN, W. P., KIRKPATRICK, B., FISCHER, B. A., KELLER, W. R., MISKI, P., BUCHANAN, R. W., GREEN, M. F. \& CARPENTER JR, W. T. 2013. Deconstructing negative symptoms of schizophrenia: avolition-apathy and diminished expression clusters predict clinical presentation and functional outcome. Journal of psychiatric research, 47, 783-790.

STRAUSS, G. P., KELLER, W. R., BUCHANAN, R. W., GOLD, J. M., FISCHER, B. A., MCMAHON, R. P., CATALANO, L. T., CULBRETH, A. J., CARPENTER, W. T. \& KIRKPATRICK, B. 2012b. Next-generation negative symptom assessment for clinical trials: validation of the Brief Negative Symptom Scale. Schizophrenia research, 142, 88-92. 
STRAUSS, G. P., NUÑEZ, A., AHMED, A. O., BARCHARD, K. A., GRANHOLM, E., KIRKPATRICK, B., GOLD, J. M. \& ALLEN, D. N. 2018. The latent structure of negative symptoms in schizophrenia. JAMA psychiatry, 75, 1271-1279.

WALTZ, J. A., FRANK, M. J., ROBINSON, B. M. \& GOLD, J. M. 2007. Selective reinforcement learning deficits in schizophrenia support predictions from computational models of striatal-cortical dysfunction. Biological psychiatry, 62, 756-764.

WALTZ, J. A., XU, Z., BROWN, E. C., RUIZ, R. R., FRANK, M. J. \& GOLD, J. M. 2018. Motivational deficits in schizophrenia are associated with reduced differentiation between gain and loss-avoidance feedback in the striatum. Biological Psychiatry: Cognitive Neuroscience and Neuroimaging, 3, 239-247. 


\section{Table 1}

Demographic, psychopathological and clinical characteristics of patients

\begin{tabular}{|c|c|c|c|}
\hline & Study $1(\mathrm{~N}=120)$ & Study $2(N=31)$ & Study $3(N=43)$ \\
\hline Age, years & $32.4(10.7)$ & $30.4(8.7)$ & $32.2(8)$ \\
\hline Sex & 87 males & 25 males & 32 males \\
\hline Education, years & $11.8(3.3)$ & $9.8(1.7)$ & $12.1(2.6)$ \\
\hline Duration of illness, months & $119.9(186.6)$ & $115.5(97.5)$ & $115.3(81.5)$ \\
\hline $\begin{array}{l}\text { Chlorpromazine equivalent, } \\
\mathrm{mg} / \mathrm{d}\end{array}$ & $585.1(562)$ & $568.2(409.97)$ & $514.2(457.26)$ \\
\hline $\begin{array}{l}\text { Calgary Depression Scale for } \\
\text { Schizophrenia }\end{array}$ & $2.24(2.9)$ & $2.42(2.41)$ & $2.15(2.15)$ \\
\hline \multicolumn{4}{|l|}{ BNSS score } \\
\hline Amotivation & $17.03(8.9)$ & $16.6(7.5)$ & $15.09(7.8)$ \\
\hline Diminished Expression & $10.3(8.3)$ & $10.4(6.97)$ & $9.07(7.6)$ \\
\hline \multicolumn{4}{|l|}{ PANSS score } \\
\hline Amotivation & $7.8(3.4)$ & $7.06(2.7)$ & $7.3(3.3)$ \\
\hline Diminished Expression & $9(4.4)$ & $9.06(3.7)$ & $8.95(4.3)$ \\
\hline \multicolumn{4}{|l|}{ SANS score } \\
\hline Amotivation & $4.4(2.2)$ & $4.03(1.8)$ & $4.5(2.2)$ \\
\hline Diminished Expression & $2.7(2.4)$ & $2.9(1.98)$ & $2.79(2.5)$ \\
\hline
\end{tabular}




\section{Table 2}

Factor solutions from the literature (Mucci et al., 2015, Fervaha et al., 2014) used to calculate Amotivation and Diminished expression factors for BNSS and PANSS. For completeness, we also show the factor solution proposed by Liemburg and colleagues (2013)

\begin{tabular}{|l|l|}
\hline \multicolumn{1}{|c|}{ Amotivation } & \multicolumn{1}{|c|}{ Diminished expression } \\
\hline \multicolumn{1}{|c|}{ BNSS (Mucci et al., 2015) } \\
\hline Intensity of pleasure & Facial expression \\
\hline Frequency of pleasure & Vocal expression \\
\hline Intensity of future pleasure & Expressive gestures \\
\hline Asociality: behaviour & Quantity of speech \\
\hline Asociality: internal experience & Spontaneous elaboration \\
\hline Avolition: behaviour & \\
\hline Avolition: internal experience & \\
\hline \multicolumn{2}{|c|}{ PANSS (Fervaha et al., 2014) } \\
\hline Emotional withdrawal (N2) & Blunted affect (N1) \\
\hline Apathetic social withdrawal (N4) & Poor rapport (N3) \\
\hline Active social avoidance (G16) & Lack of flow (N6) \\
\hline \multicolumn{2}{|c|}{ PANSS (Liemburg et al. 2013) } \\
\hline \multicolumn{2}{|c|}{ Motor retardation (G7) } \\
\hline Emotional withdrawal (N2) & Blunted affect (N1) \\
\hline Apathetic social withdrawal (N4) & Poor rapport (N3) \\
\hline Active social avoidance (G16) & Lack of flow (N6) \\
\hline & Motor retardation (G7) \\
\hline & Mannerisms and posturing (G5) \\
\hline & Avolition (G13) \\
\hline
\end{tabular}


Table 3

Correlation matrix for BNSS and PANSS $(\mathrm{N}=120)$

\begin{tabular}{|c|c|c|c|c|c|}
\hline & & $\begin{array}{c}\text { BNSS } \\
\text { Amotivation }\end{array}$ & $\begin{array}{c}\text { BNSS } \\
\text { Expression }\end{array}$ & $\begin{array}{c}\text { PANSS } \\
\text { Amotivation }\end{array}$ & $\begin{array}{c}\text { PANSS } \\
\text { Expression }\end{array}$ \\
\hline \multirow[t]{2}{*}{$\begin{array}{l}\text { BNSS } \\
\text { Amotivation }\end{array}$} & $\begin{array}{l}\text { Pearson's } \\
r\end{array}$ & - & 0.651 & 0.830 & 0.633 \\
\hline & p-value & - & $<.001$ & $<.001$ & $<.001$ \\
\hline \multirow[t]{2}{*}{ BNSS Expression } & $\begin{array}{l}\text { Pearson's } \\
r\end{array}$ & & - & 0.665 & 0.909 \\
\hline & $\mathrm{p}$-value & & - & $<.001$ & $<.001$ \\
\hline \multirow[t]{2}{*}{$\begin{array}{l}\text { PANSS } \\
\text { Amotivation }\end{array}$} & $\begin{array}{l}\text { Pearson's } \\
r\end{array}$ & & & 一 & 0.684 \\
\hline & $\mathrm{p}$-value & & & - & $<.001$ \\
\hline \multirow[t]{2}{*}{$\begin{array}{l}\text { PANSS } \\
\text { Expression }\end{array}$} & $\begin{array}{l}\text { Pearson's } \\
r\end{array}$ & & & & - \\
\hline & $p$-value & & & & - \\
\hline
\end{tabular}


Table 4

Correlation Matrix between PANSS Amotivation and BNSS Anhedonia, Avolition and Asociality $(\mathrm{N}=120)$

\begin{tabular}{llr}
\hline & & \multicolumn{1}{c}{$\begin{array}{c}\text { PANSS } \\
\text { Amotivation }\end{array}$} \\
\hline PANSS Amotivation & Pearson's $r$ & - \\
p-value & 0.696 \\
BNSS Anhedonia & Pearson's $r$ & $<.001$ \\
BNSS Asociality & p-value & 0.791 \\
BNSS Avolition & Pearson's $r$ & $<.001$ \\
& p-value & 0.765 \\
& Pearson's $r$ & $<.001$ \\
\hline
\end{tabular}


Table 5

Correlation matrix between PANSS Diminished Expression and BNSS Blunted affect and Alogia ( $N=120)$

PANSS

Expression

$\begin{array}{llr}\text { PANSS Expression } & \text { Pearson's } r & - \\ & \text { p-value } & - \\ \text { BNSS Blunted affect } & \text { Pearson's } r & 0.867 \\ & \text { p-value } & <.001 \\ \text { BNSS Alogia } & \text { Pearson's } r & 0.885 \\ & \text { p-value } & <.001\end{array}$

Table 6

Correlation Matrix for BNSS, PANSS and area under the curve (AUC) reflecting willingness to exert effort ( $N=31$, Hartmann et al., 2015)

\begin{tabular}{lll} 
& & AUC \\
\hline PANSS Amotivation & Pearson's $r$ & -0.492 \\
& p-value & 0.005 \\
PANSS Expression & Pearson's $r$ & -0.077 \\
& p-value & 0.682 \\
BNSS Amotivation & Pearson's r & -0.665 \\
& p-value & $<.001$ \\
BNSS Expression & Pearson's $r$ & -0.143 \\
& p-value & 0.444 \\
\hline
\end{tabular}


Table 7

Correlation Matrix between BNSS, PANSS and the left ventral striatum activation reflecting reward anticipation ( $\mathrm{N}=41$, Kirschner et al., 2016, Stepien et al., 2018)

left VS

\begin{tabular}{lll}
\hline BNSS Amotivation & Pearson's r & -0.523 \\
& p-value & $<.001$ \\
BNSS Expression & Pearson's r & 0.042 \\
& p-value & 0.794 \\
PANSS Amotivation & Pearson's r & -0.326 \\
& p-value & 0.037 \\
PANSS Expression & Pearson's r & 0.007 \\
& p-value & 0.966 \\
\hline
\end{tabular}


A

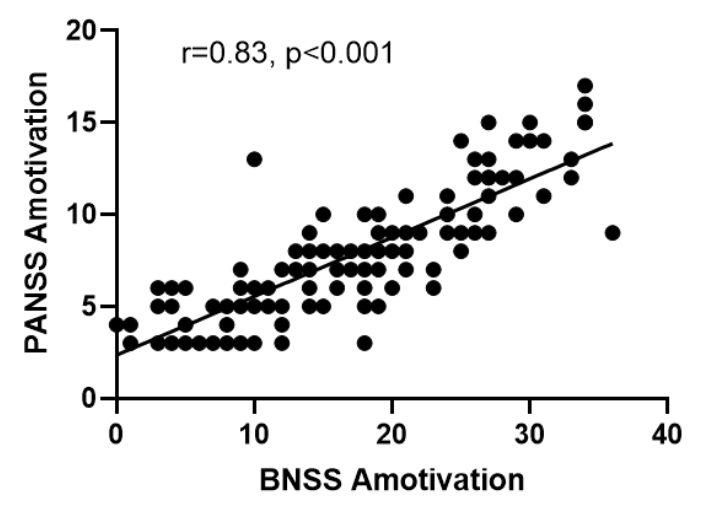

C

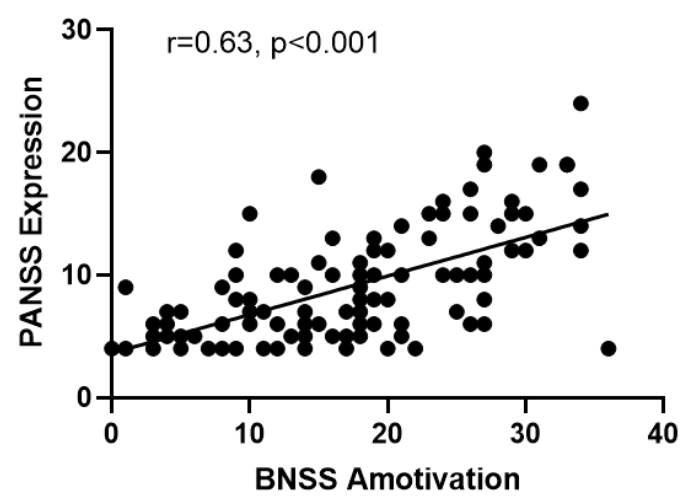

B

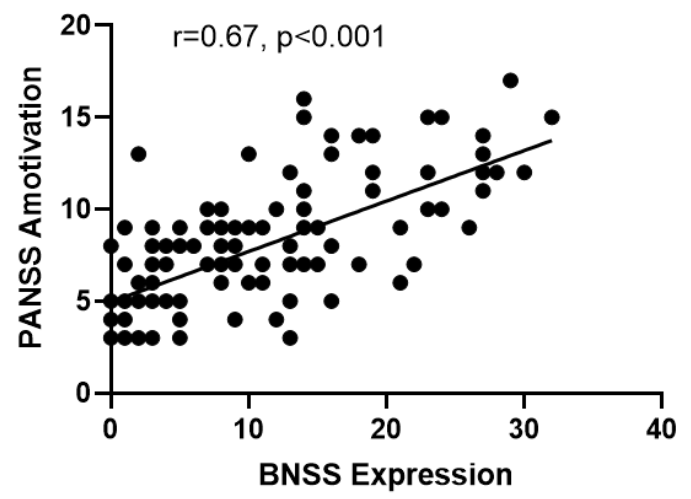

D

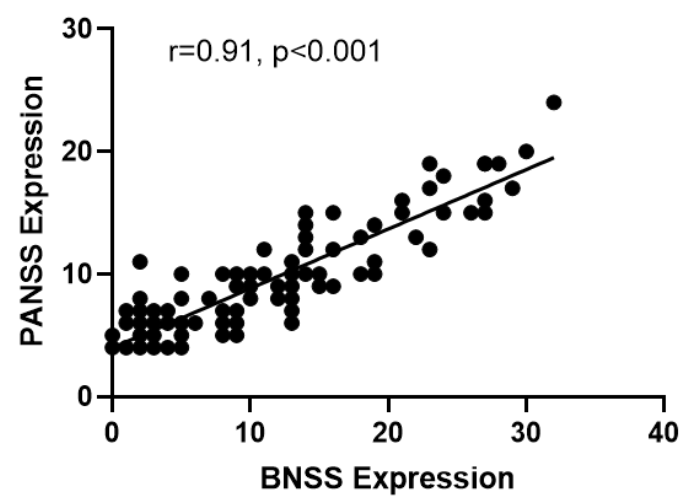

Figure 1. Pearson correlation for the factors Amotivation and Diminished expression of the BNSS and the PANSS factors (A,D - correlations within factors; $B, C$ - correlation across factors). 
A

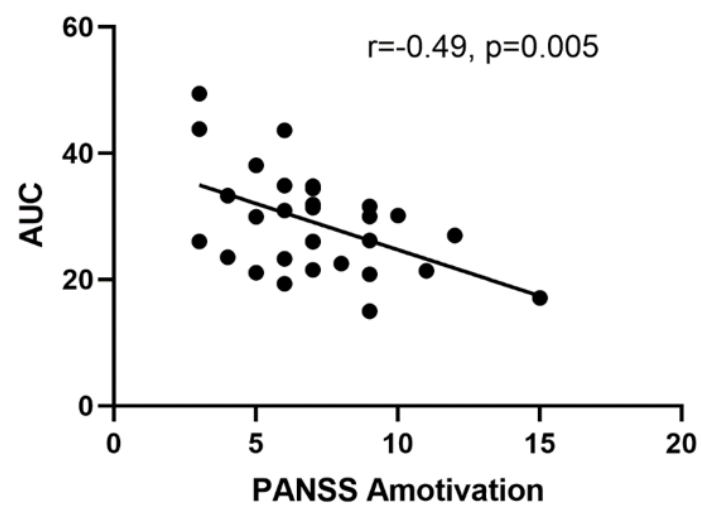

B

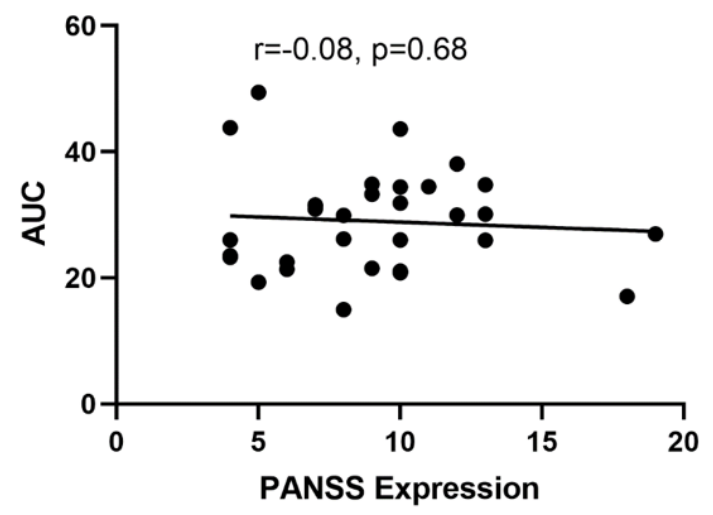

Figure 2. Pearson correlations of the PANSS factors Amotivation (A) and Diminished expression (B) with the willingness to exert effort (AUC).

A

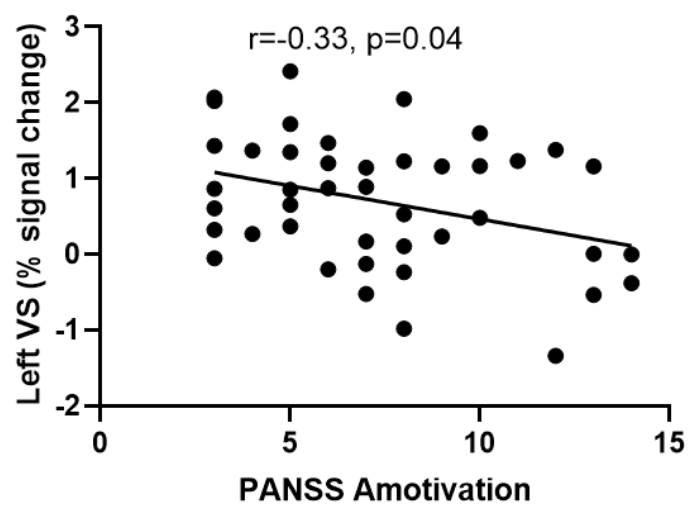

B

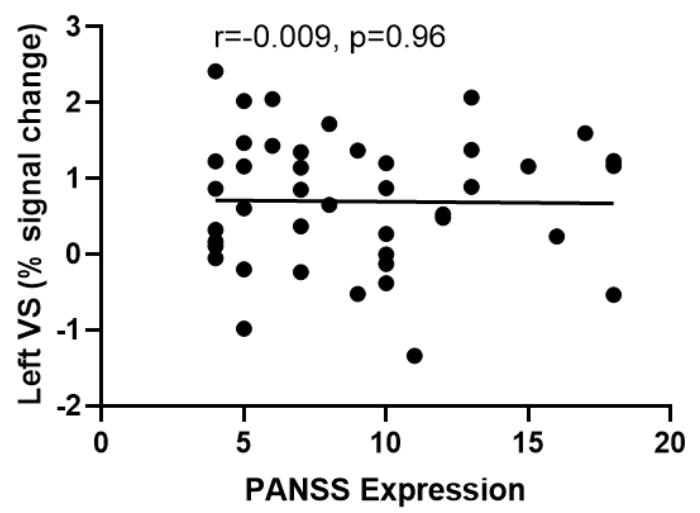

Figure 3. Pearson correlation of the factors Amotivation (A) and Diminished expression (B) with percent signal change reflecting reward anticipation in the left ventral striatum. 


\section{Supplementary material}

\section{Clinical validation of the SANS}

All the analyses described in the main text of the article were repeated using the two factors of the SANS (Table S1).

\section{Study 1 - Clinical Validation}

Comparing PANSS factors to SANS factors

We compared the PANSS factors to the SANS factors for further validation. SANS data were available from 75 participants of the original sample (Bischof et al., 2016); analyses were thus performed on data from these participants for all scales.

As expected, we found that the PANSS amotivation factor was highly correlated with the SANS amotivation factor (Table S2). Importantly, this correlation was significantly stronger than the correlation between the PANSS amotivation factor and the SANS diminished expression score $(z=2.5, p=0.014)$. Furthermore, the PANSS diminished expression factor was highly correlated with the SANS diminished expression factor and this correlation was significantly stronger than the correlation between the PANSS diminished expression factor and the SANS amotivation factor $(z=4.9, p<0.0001)$.

The correlation matrix between the BNSS, PANSS and SANS confirmed the strong correlations for the factors amotivation and diminished expression between BNSS and PANSS shown in the main text of the manuscript (Table S2).

\section{Study 2 - Behavioural validation}

Similarly to BNSS and PANSS, the negative correlation of AUC was significant for SANS amotivation ( $r=-$ $0.58, p<0.001)$ but not for SANS diminished expression $(r=-0.17, p=0.37)$. There was no difference between the correlations of AUC with BNSS amotivation and SANS amotivation $(z=-1.34, p=0.18)$, as well as between the correlation of AUC with PANSS amotivation and SANS amotivation $(z=0.98, p=0.33)$ (Table S3).

\section{Study 3 - Neural validation}

The SANS factors show a similar pattern of results as the BNSS and the PANSS factors (Table S4). Specifically, the strength of the association of ventral striatum signal change with SANS amotivation did not differ significantly from that with BNSS amotivation ( $z=0.07, p=0.9)$.

The three analyses show better correspondence between BNSS and SANS than between BNSS and PANSS. The preference of SANS over PANSS for the assessment of negative symptoms has previously been acknowledged, in that the SANS better reflects the defined domains of negative symptoms and uses several items to assess each domain (Kirkpatrick et al., 2006). However, our results show that all the three scales allow the distinction between the factors amotivation and diminished expression and that either 
the SANS or the PANSS could thus be used in studies where only a restricted number of scales can be employed. 
Supplementary tables

Table S1. Factor solutions from the literature used to calculate Amotivation and Diminished expression factors for the SANS

\begin{tabular}{|c|c|}
\hline Amotivation & Diminished expression \\
\hline \multicolumn{2}{|c|}{ SANS (Strauss et al., 2013) } \\
\hline Avolition & Affective flattening \\
\hline Anhedonia-Asociality & Alogia \\
\hline
\end{tabular}

Table S2. Correlation Matrix for BNSS, PANSS and SANS (75 participants)

\begin{tabular}{|c|c|c|c|c|c|c|c|}
\hline & & $\begin{array}{c}\text { BNSS } \\
\text { Amotivatio } \\
\mathbf{n}\end{array}$ & $\begin{array}{c}\text { BNSS } \\
\text { Expressio } \\
\mathbf{n}\end{array}$ & $\begin{array}{c}\text { PANSS } \\
\text { Amotivatio } \\
\mathbf{n}\end{array}$ & $\begin{array}{c}\text { PANSS } \\
\text { Expressio } \\
\mathbf{n}\end{array}$ & $\begin{array}{c}\text { SANS } \\
\text { Amotivatio } \\
\mathbf{n}\end{array}$ & $\begin{array}{c}\text { SANS } \\
\text { Expressio } \\
\mathbf{n}\end{array}$ \\
\hline \multirow[t]{2}{*}{$\begin{array}{l}\text { BNSS } \\
\text { Amotivatio } \\
n\end{array}$} & $\begin{array}{l}\text { Pearson' } \\
\text { s r }\end{array}$ & - & 0.542 & 0.773 & 0.529 & 0.815 & 0.544 \\
\hline & $\mathrm{p}$-value & - & $<.001$ & $<.001$ & $<.001$ & $<.001$ & $<.001$ \\
\hline \multirow[t]{2}{*}{$\begin{array}{l}\text { BNSS } \\
\text { Expression }\end{array}$} & $\begin{array}{l}\text { Pearson' } \\
\text { s r }\end{array}$ & & - & 0.650 & 0.876 & 0.545 & 0.927 \\
\hline & $\mathrm{p}$-value & & - & $<.001$ & $<.001$ & $<.001$ & $<.001$ \\
\hline \multirow[t]{2}{*}{$\begin{array}{l}\text { PANSS } \\
\text { Amotivatio } \\
n\end{array}$} & $\begin{array}{l}\text { Pearson' } \\
\text { s r }\end{array}$ & & & - & 0.672 & 0.811 & 0.649 \\
\hline & $p$-value & & & - & $<.001$ & $<.001$ & $<.001$ \\
\hline \multirow[t]{2}{*}{$\begin{array}{l}\text { PANSS } \\
\text { Expression }\end{array}$} & $\begin{array}{l}\text { Pearson' } \\
\text { s r }\end{array}$ & & & & - & 0.548 & 0.915 \\
\hline & $p$-value & & & & - & $<.001$ & $<.001$ \\
\hline \multirow[t]{2}{*}{$\begin{array}{l}\text { SANS } \\
\text { Amotivatio } \\
n\end{array}$} & $\begin{array}{l}\text { Pearson' } \\
\mathrm{sr}\end{array}$ & & & & & - & 0.577 \\
\hline & $\mathrm{p}$-value & & & & & - & $<.001$ \\
\hline \multirow[t]{2}{*}{$\begin{array}{l}\text { SANS } \\
\text { Expression }\end{array}$} & $\begin{array}{l}\text { Pearson' } \\
\text { s r }\end{array}$ & & & & & & - \\
\hline & $p$-value & & & & & & - \\
\hline
\end{tabular}


Table S3. Correlation Matrix for SANS and area under the curve (AUC) reflecting willingness to exert effort $(\mathbf{N}=31)$

\begin{tabular}{llr} 
& & AUC \\
\hline \multirow{2}{*}{ AUC } & Pearson's $r$ & - \\
& p-value & - \\
SANS Expression & Pearson's $r$ & -0.169 \\
& p-value & 0.365 \\
SANS Amotivation & Pearson's $r$ & -0.577 \\
& p-value & $<.001$ \\
\hline
\end{tabular}

Table S4. Correlation Matrix for SANS and left ventral striatum activation $(\mathbf{N}=\mathbf{4 1})$

\begin{tabular}{|c|c|c|}
\hline & & left VS \\
\hline \multirow[t]{2}{*}{ left VS } & $\begin{array}{l}\text { Pearson's } \\
r\end{array}$ & - \\
\hline & $p$-value & - \\
\hline \multirow[t]{2}{*}{ SANS Amotivation } & $\begin{array}{l}\text { Pearson's } \\
r\end{array}$ & -0.533 \\
\hline & $p$-value & $<.001$ \\
\hline \multirow[t]{2}{*}{ SANS Expression } & $\begin{array}{l}\text { Pearson's } \\
r\end{array}$ & -0.111 \\
\hline & p-value & 0.495 \\
\hline
\end{tabular}




\section{Clinical validation of the PANSS}

In an additional analysis, we included only the 75 patients which exclusively participated in Study 1 and not in Study 2 or 3 in order assure full independence. The results for this subgroup were almost identical to the full sample (see supplementary material, and supplementary table S1).

First, the PANSS amotivation factor was highly correlated with the BNSS amotivation factor. Similarly to the main result in the full 120 sample, this correlation was significantly stronger than the correlation between the PANSS amotivation factor and the BNSS diminished expression score $(z=2.8, p<0.005)$. The same was true for the diminished expression factor: the PANSS diminished expression factor was highly correlated with the BNSS diminished expression factor and this correlation was significantly stronger than the correlation between the PANSS diminished expression factor and the BNSS amotivation factor ( $z=4.02$, $\mathrm{p}=0.0001$ ).

Table S5. Correlation Matrix for BNSS \& PANSS factors (75 participants)

\section{PANSS Amotivation PANSS Expression BNSS Amotivation}

\begin{tabular}{llrrr}
\hline PANSS Amotivation & Pearson's r & - & \\
& p-value & - & - & \\
PANSS Expression & Pearson's r & 0.732 & - & - \\
& p-value & $<.001$ & 0.696 & - \\
BNSS Amotivation & Pearson's r & 0.863 & $<.001$ & 0.711 \\
& p-value & $<.001$ & 0.915 & $<.001$ \\
\hline \multirow{2}{*}{ BNSS Expression } & Pearson's $r$ & 0.722 & $<.001$ & \\
& p-value & $<.001$ & & - \\
\hline
\end{tabular}

